Pathophysiology of Haemostasis and Thrombosis

\title{
Common C677T Polymorphism of the Methylenetetrahydrofolate Reductase Gene and the Risk of Venous Thromboembolism: Meta-Analysis of 31 Studies
}

\author{
J.G. Ray ${ }^{a, b}$ D. Shmorgun ${ }^{b}$ W.S. Chan ${ }^{a}$
}

aDepartment of Medicine, Women's College Campus, Sunnybrook and Women's College Health Sciences Centre, Toronto, Ont., bMcMaster University, Hamilton, Ont., Canada

\author{
Key Words \\ Venous thromboembolism - Deep vein thrombosis . \\ Pulmonary embolism . Homocysteine. \\ Methylenetetrahydrofolate reductase . Thrombophilia . \\ Genetics · Meta-analysis
}

\begin{abstract}
Background: Although the common $677 \mathrm{C} \rightarrow \mathrm{T}$ polymorphism of the methylenetetrahydrofolate reductase (MTHFR) gene is implicated in the development of hyperhomocysteinemia, its correlation with venous thromboembolism (VTE) remains controversial. We conducted a meta-analysis of previously published studies to clarify the role of the MTHFR 677 TT homozygous genotype in association with VTE. Methods and Results: Relevant articles were retrieved from a systematic search of Medline and Embase from 1990 to September 2001. Two independent reviewers abstracted data on the characteristics of the cases with VTE and controls without VTE. We pooled the rates of the MTHFR 677 TT genotype in both groups, as well as the odds ratio (OR) of VTE in the presence of the TT versus CC or CT genotypes. In 31 published studies, comprising 4,901 cases and 7,886 controls, the pooled prevalence of the MTHFR 677 TT genotype was slightly higher among cases $(14.3 \%)$ than controls $(11.7 \%)$, conferring a borderline degree of heightened risk [pooled $\mathrm{OR}\left(\mathrm{OR}_{\mathrm{p}}\right)$ 1.2, 95\% confidence interval
\end{abstract}

(Cl) 1.1-1.4]. After excluding cases with a classic thrombophilia factor, the pooled prevalence rates of the MTHFR 677 TT genotype among 11 studies were 17.7 and $12.3 \%$, respectively $\left(\mathrm{OR}_{\mathrm{p}} 1.5,95 \% \mathrm{Cl} 1.2-1.9\right)$. Conclusions: The classic MTHFR C677T gene polymorphism is weakly associated with an increased risk of VTE. It is unlikely that the purported relationship between hyperhomocysteinemia and VTE is mediated by this gene defect to a substantial degree, although other undiscovered gene polymorphisms may explain this association. Until more compelling data are made available, we do not recommend that testing for the MTHFR C67TT polymorphism be routinely included as part of any clinical thrombophilia assessment.

Copyright @2002 S. Karger AG, Basel

\section{Introduction}

Elevated plasma homocysteine (tHcy) has been associated with an increased risk of venous thromboembolism (VTE) [1]. Hyperhomocysteinemia may result from inadequate folic acid or cobalamin stores, renal insufficiency or one of several gene-based mechanisms, including the common thermolabile variant of 5,10-methylenetetrahydrofolate reductase (MTHFR), an enzyme that provides the folate derivative for remethylation of tHcy to methionine $[2,3]$. The enzyme defect often results from a mis-

\begin{tabular}{ll}
\hline KARGER & ( ) 2002 S. Karger AG, Basel \\
1424-8832/02/0322-0051\$18.50/0 \\
$\begin{array}{l}\text { Fax +4161306 1234 } \\
\begin{array}{l}\text { E-Mail karger@karger.ch } \\
\text { www.karger.com }\end{array}\end{array}$ & $\begin{array}{l}\text { Accessible online at: } \\
\text { www.karger.com/journals/pht }\end{array}$
\end{tabular}

Dr. J.G. Ray

E-Mail rayjg@mcmaster.ca 
sense mutation at base pair $677 \mathrm{C} \rightarrow \mathrm{T}$ of MTHFR, which, in its homozygous (TT) state, leads to the phenotypic expression of the enzyme defect [3].

Although hyperhomocysteinemia may be an independent risk factor for cardiovascular disease [4, 5], a metaanalysis of 23 case-control studies failed to show a direct association between MTHFR C677T homozygosity and cardiovascular disease [odds ratio (OR) 1.1, 95\% confidence interval (CI) 0.9-1.4] [6]. Similarly, there have been conflicting opinions about the importance of the MTHFR C677T polymorphism as a risk factor for VTE $[7,8]$. To clarify the thrombophilic role of MTHFR C677T, we conducted a meta-analysis of published studies examining the association between the MTHFR C677T homozygous genotype and VTE.

\section{Methods}

Literature Search

Two authors (D.S. and J.G.R.) searched both PubMed Medline and Embase from 1990 to September 2001, merging the following textword and MeSH headings: ('MTHFR' or 'methylenetetrahydrofolate reductase') and ('deep vein thrombosis', 'venous thromboembolism', 'venous thrombosis', 'pulmonary embolism' or 'thrombophlebitis'). We also examined the references of all studies for other potential citations, but did not search for unpublished studies. English-language reports that met all of the following criteria were included: (1) use of a cohort or case-control design; (2) cases were aged 16 years and older; (3) at least 20 cases were included, and (4) the presence of the MTHFR C677 gene polymorphism was assessed both among subjects with (i.e. cases) and without (i.e. controls) VTE. In those situations in which it was unclear whether cases had been used in more than one published study, we contacted the principal author by E-mail to ask for clarification.

Table 1. Characteristics of study participants

\begin{tabular}{|c|c|c|c|c|c|c|c|c|c|c|}
\hline \multirow[t]{2}{*}{ Study } & \multicolumn{7}{|l|}{ Cases with VTE } & \multicolumn{3}{|l|}{ Controls without VTE } \\
\hline & source and definition & $\begin{array}{l}\text { excluded } \\
\text { those } \\
\text { cancer or } \\
\text { situational } \\
\text { VTE? }\end{array}$ & $\begin{array}{l}\text { excluded } \\
\text { those with } \\
\text { a 'classic' } \\
\text { thrombophilia } \\
\text { marker?' }\end{array}$ & $\begin{array}{l}\text { number } \\
\text { of males }\end{array}$ & $\begin{array}{l}\text { mean/ } \\
\text { median } \\
\text { age } \\
\text { years }\end{array}$ & $\begin{array}{l}\text { VTE } \\
\text { type }\end{array}$ & $\begin{array}{l}\text { objective } \\
\text { assessment } \\
\text { of VTE? }\end{array}$ & source and definition & $\begin{array}{l}\text { number } \\
\text { of males }\end{array}$ & $\begin{array}{l}\text { mean/ } \\
\text { median } \\
\text { age } \\
\text { years }\end{array}$ \\
\hline $\begin{array}{l}\text { Arruda } \\
\text { et al. } 1997[7]\end{array}$ & $\begin{array}{l}\text { Brazilian outpatients } \\
\text { with VTE }\end{array}$ & yes & yes & $51(40)$ & 35 & $\begin{array}{l}\text { DVT } \\
\text { or PE }\end{array}$ & yes & $\begin{array}{l}\text { consecutive newborns delivered } \\
\text { at same hospital }\end{array}$ & NA & $\begin{array}{l}\text { new- } \\
\text { born }\end{array}$ \\
\hline $\begin{array}{l}\text { Cattaneo } \\
\text { et al. } 1997[12]\end{array}$ & $\begin{array}{l}\text { Italian patients with } \\
\text { DVT }\end{array}$ & yes & no & $35(45)$ & 39 & $\begin{array}{l}\text { DVT } \\
\text { or PE }\end{array}$ & yes & $\begin{array}{l}\text { healthy adults without a history of } \\
\text { VTE or arterial occlusive disease }\end{array}$ & $70(45)$ & 43 \\
\hline $\begin{array}{l}\text { Ocal } \\
\text { et al. } 1997 \text { [13] }\end{array}$ & $\begin{array}{l}\text { US patients with VTE } \\
\text { and available blood } \\
\text { samples }\end{array}$ & no & no & $145(44)$ & 44 & $\begin{array}{l}\text { DVT } \\
\text { or PE }\end{array}$ & NA & healthy blood donors & $84(50)$ & 46 \\
\hline $\begin{array}{l}\text { Salden } \\
\text { et al. } 1997[14]\end{array}$ & UK patients with VTE & yes & yes & NA & 43 & $\begin{array}{l}\text { DVT } \\
\text { or PE }\end{array}$ & yes & healthy plasma donors & NA & 34 \\
\hline $\begin{array}{l}\text { Tosetto } \\
\text { et al. } 1997[15]\end{array}$ & $\begin{array}{l}\text { Italian patients with } \\
\text { VTE }\end{array}$ & no & no & $21(32)$ & 52 & $\begin{array}{l}\text { DVT } \\
\text { or PE }\end{array}$ & yes & $\begin{array}{l}\text { individuals randomly selected } \\
\text { from the population without a } \\
\text { history of VTE }\end{array}$ & $42(32)$ & 51 \\
\hline $\begin{array}{l}\text { Brown } \\
\text { et al. } 1998[16]\end{array}$ & UK patients with VTE & no & no & NA & 45 & VTE & yes & $\begin{array}{l}\text { blood donors from same geo- } \\
\text { graphic area as cases }\end{array}$ & NA & 38 \\
\hline $\begin{array}{l}\text { Dilley } \\
\text { et al. } 1998[17]\end{array}$ & $\begin{array}{l}\text { African-American out- } \\
\text { patients with VTE }\end{array}$ & no & no & $39(42)$ & 55 & $\begin{array}{l}\text { DVT } \\
\text { or PE }\end{array}$ & yes & $\begin{array}{l}\text { African-American outpatients un- } \\
\text { dergoing routine laboratory tests } \\
\text { without a history of VTE or CVD }\end{array}$ & $91(49)$ & 56 \\
\hline $\begin{array}{l}\text { Grandone } \\
\text { et al. } 1998[18]\end{array}$ & $\begin{array}{l}\text { Italian women with } \\
\text { VTE during pregnancy } \\
\text { or puerperium }\end{array}$ & no & no & $0(0)$ & 36 & $\begin{array}{l}\text { DVT } \\
\text { or PE }\end{array}$ & yes & $\begin{array}{l}\text { parous women without a history } \\
\text { of VTE or arterial thrombosis }\end{array}$ & $0(0)$ & 34 \\
\hline $\begin{array}{l}\text { Kluijtmans } \\
\text { et al. } 1998 \text { [19] }\end{array}$ & $\begin{array}{l}\text { Dutch anticoagulant } \\
\text { clinic patients with } \\
\text { first DVT }\end{array}$ & no & no & $205(44)$ & 44 & DVT & yes & $\begin{array}{l}\text { individuals without a history of } \\
\text { VTE }\end{array}$ & $206(44)$ & 44 \\
\hline $\begin{array}{l}\text { Margaglione } \\
\text { et al. } 1998[20]\end{array}$ & $\begin{array}{l}\text { Italian patients with } \\
\text { first VTE }\end{array}$ & yes & yes & $98(44)$ & 38 & $\begin{array}{l}\text { DVT } \\
\text { or PE }\end{array}$ & yes & $\begin{array}{l}\text { healthy individuals without a } \\
\text { history of VTE }\end{array}$ & $244(56)$ & 36 \\
\hline $\begin{array}{l}\text { Rintelen } \\
\text { et al. } 1999 \text { [21] }\end{array}$ & $\begin{array}{l}\text { Austrian patients with } \\
\text { VTE }\end{array}$ & yes & yes & $27(3)$ & 41 & $\begin{array}{l}\text { DVT } \\
\text { or PE }\end{array}$ & yes & $\begin{array}{l}\text { hospital staff without a history } \\
\text { of VTE or arterial thrombosis }\end{array}$ & NA & 36 \\
\hline $\begin{array}{l}\text { Alhenc-Gelas } \\
\text { et al. } 1999 \text { [22] }\end{array}$ & $\begin{array}{l}\text { French patients aged } \\
\leq 60 \text { years with } \\
\geq 1 \text { VTE events }\end{array}$ & no & no & $92(45)$ & 42 & $\begin{array}{l}\text { DVT } \\
\text { or PE }\end{array}$ & yes & $\begin{array}{l}\text { healthy individuals for routine } \\
\text { checkup without a history of VTE }\end{array}$ & $199(50)$ & 43 \\
\hline
\end{tabular}


Table 1 (continued)

\begin{tabular}{|c|c|c|c|c|c|c|c|c|c|c|}
\hline \multirow[t]{2}{*}{ Study } & \multicolumn{7}{|l|}{ Cases with VTE } & \multicolumn{3}{|l|}{ Controls without VTE } \\
\hline & source and definition & $\begin{array}{l}\text { excluded } \\
\text { those } \\
\text { cancer or } \\
\text { situational } \\
\text { VTE? }\end{array}$ & $\begin{array}{l}\text { excluded } \\
\text { those with } \\
\text { a 'classic' } \\
\text { thrombophilia } \\
\text { marker?' }\end{array}$ & $\begin{array}{l}\text { number } \\
\text { of males }\end{array}$ & $\begin{array}{l}\text { mean/ } \\
\text { median } \\
\text { age } \\
\text { years }\end{array}$ & $\begin{array}{l}\text { VTE } \\
\text { type }\end{array}$ & $\begin{array}{l}\text { objective } \\
\text { assessment } \\
\text { of VTE? }\end{array}$ & source and definition & $\begin{array}{l}\text { number } \\
\text { of males }\end{array}$ & $\begin{array}{l}\text { mean/ } \\
\text { median } \\
\text { age } \\
\text { years }\end{array}$ \\
\hline $\begin{array}{l}\text { Cattaneo } \\
\text { et al. } 1999[23]\end{array}$ & $\begin{array}{l}\text { Italian patients with } \\
\text { first DVT }\end{array}$ & yes & no & $45(38)$ & 39 & DVT & yes & $\begin{array}{l}\text { healthy individuals without a } \\
\text { history of VTE }\end{array}$ & $164(39)$ & 43 \\
\hline $\begin{array}{l}\text { Ho } \\
2000[24]\end{array}$ & $\begin{array}{l}\text { Taiwanese Chinese } \\
\text { with VTE }\end{array}$ & no & no & $28(57)$ & 58 & NA & yes & $\begin{array}{l}\text { individuals presenting for a rou- } \\
\text { tine checkup or outpatient blood } \\
\text { work, without a history of VTE }\end{array}$ & $830(70)$ & 48 \\
\hline $\begin{array}{l}\text { Franco } \\
\text { et al. } 1999[25]\end{array}$ & $\begin{array}{l}\text { Brazilian patients aged } \\
\leq 65 \text { years with DVT }\end{array}$ & yes & no & $72(38)$ & 41 & DVT & yes & $\begin{array}{l}\text { healthy blood donors without a } \\
\text { personal or family history of VTE }\end{array}$ & $72(38)$ & 41 \\
\hline $\begin{array}{l}\text { Gemmati } \\
\text { et al. } 1999 \text { [26] }\end{array}$ & $\begin{array}{l}\text { Italian patients aged } \\
\leq 75 \text { years with } \\
\geq 1 \text { VTE event }\end{array}$ & yes & yes & $124(56)$ & 54 & $\begin{array}{l}\text { DVT } \\
\text { or PE }\end{array}$ & yes & $\begin{array}{l}\text { blood donors without a personal } \\
\text { or family history of VTE }\end{array}$ & $135(61)$ & 46 \\
\hline $\begin{array}{l}\text { Gemmati } \\
\text { et al. } 1999 \text { [27] }\end{array}$ & $\begin{array}{l}\text { Italian patients aged } \\
\leq 65 \text { years with } \\
\geq 1 \text { VTE event }\end{array}$ & yes & yes & $89(49)$ & 50 & $\begin{array}{l}\text { DVT } \\
\text { or PE }\end{array}$ & yes & $\begin{array}{l}\text { blood donors without a personal } \\
\text { or family history of VTE }\end{array}$ & $100(50)$ & 49 \\
\hline $\begin{array}{l}\text { Salomon } \\
\text { et al. } 1999 \text { [28] }\end{array}$ & $\begin{array}{l}\text { Israeli patients with } \\
\geq 1 \text { VTE event }\end{array}$ & yes & yes & $67(41)$ & 42 & $\begin{array}{l}\text { DVT } \\
\text { or PE }\end{array}$ & yes & $\begin{array}{l}\text { patients consecutively admitted to } \\
\text { hospital without a history of VTE }\end{array}$ & $153(46)$ & 71 \\
\hline $\begin{array}{l}\text { Lin } \\
\text { et al. } 2000 \text { [29] }\end{array}$ & $\begin{array}{l}\text { Chinese Taiwanese } \\
\text { patients with VTE }\end{array}$ & yes & yes & $56(50)$ & 48 & $\begin{array}{l}\text { DVT } \\
\text { or PE }\end{array}$ & yes & $\begin{array}{l}\text { healthy individuals without a } \\
\text { history of VTE }\end{array}$ & $67(54)$ & 46 \\
\hline $\begin{array}{l}\text { Akar } \\
\text { et al. } 2000[30]\end{array}$ & $\begin{array}{l}\text { Turkish patients with } \\
\text { DVT and no athero- } \\
\text { thrombosis }\end{array}$ & no & no & NA & NA & DVT & yes & $\begin{array}{l}\text { individuals without a personal or } \\
\text { family history of VTE or athero- } \\
\text { thrombosis }\end{array}$ & NA & NA \\
\hline $\begin{array}{l}\text { Angchaisuksiri } \\
\text { et al. } 2000[31]\end{array}$ & $\begin{array}{l}\text { Thai patients with } \\
\text { VTE }\end{array}$ & no & no & $18(45)$ & 38 & $\begin{array}{l}\text { DVT } \\
\text { or PE }\end{array}$ & yes & healthy adults & NA & NA \\
\hline $\begin{array}{l}\text { Couturaud } \\
\text { et al. } 2000 \text { [32] }\end{array}$ & $\begin{array}{l}\text { French patients with } \\
\text { VTE }\end{array}$ & yes & no & $171(47)$ & 65 & $\begin{array}{l}\text { DVT } \\
\text { or PE }\end{array}$ & yes & volunteer blood donors & NA & NA \\
\hline $\begin{array}{l}\text { De Franchis } \\
\text { et al. } 2000 \text { [33] }\end{array}$ & $\begin{array}{l}\text { Italian patients aged } \\
<45 \text { years with VTE }\end{array}$ & yes & yes & $90(47)$ & 45 & $\begin{array}{l}\text { DVT } \\
\text { or PE }\end{array}$ & yes & $\begin{array}{l}\text { healthy individuals aged }<45 \\
\text { years without a history of VTE }\end{array}$ & $101(50)$ & 41 \\
\hline $\begin{array}{l}\text { Fujimara } \\
\text { et al. } 2000 \text { [34] }\end{array}$ & $\begin{array}{l}\text { Japanese outpatients } \\
\text { with VTE }\end{array}$ & no & yes & $28(39)$ & 56 & $\begin{array}{l}\text { DVT } \\
\text { or PE }\end{array}$ & yes & $\begin{array}{l}\text { outpatients attending a venous } \\
\text { clinic for management of varicose } \\
\text { veins, without sonographic } \\
\text { evidence of DVT }\end{array}$ & $32(38)$ & 53 \\
\hline $\begin{array}{l}\text { Gerhardt } \\
\text { et al. } 2000 \text { [35] }\end{array}$ & $\begin{array}{l}\text { German women with } \\
\text { VTE during pregnancy } \\
\text { or puerperium }\end{array}$ & yes & no & $0(0)$ & 34 & $\begin{array}{l}\text { DVT } \\
\text { or PE }\end{array}$ & yes & $\begin{array}{l}\text { female blood donors, previously } \\
\text { pregnant, without a history of } \\
\text { VTE }\end{array}$ & $0(0)$ & 34 \\
\hline $\begin{array}{l}\text { Toydemir } \\
\text { et al. } 2000[36]\end{array}$ & $\begin{array}{l}\text { Turkish patients with } \\
\text { VTE }\end{array}$ & no & no & $20(67)$ & 51 & DVT & yes & $\begin{array}{l}\text { healthy individuals without a his- } \\
\text { tory of VTE or arterial thrombosis }\end{array}$ & $37(37)$ & 32 \\
\hline $\begin{array}{l}\text { Zheng } \\
\text { et al. } 2000 \text { [37] }\end{array}$ & $\begin{array}{l}\text { Chinese patients with } \\
\text { DVT }\end{array}$ & no & yes & $33(62)$ & 44 & DVT & yes & $\begin{array}{l}\text { hospital blood donors and staff } \\
\text { members }\end{array}$ & $64(52)$ & 52 \\
\hline $\begin{array}{l}\text { Guedon } \\
\text { et al. } 2001[38]\end{array}$ & $\begin{array}{l}\text { French patients with } \\
\text { VTE and no inflamma- } \\
\text { tory bowel disease }\end{array}$ & no & no & $32(29)$ & 49 & NA & NA & $\begin{array}{l}\text { individuals without a personal } \\
\text { or family history of thrombosis or } \\
\text { inflammatory bowel disease }\end{array}$ & $34(41)$ & 36 \\
\hline $\begin{array}{l}\text { Hanson } \\
\text { et al. 2001 [39] }\end{array}$ & US patients with DVT & no & no & $66(48)$ & 44 & DVT & NA & $\begin{array}{l}\text { employees of a university medical } \\
\text { center without a history of VTE } \\
\text { or CHD }\end{array}$ & $205(62)$ & 49 \\
\hline $\begin{array}{l}\text { Hsu } \\
\text { et al. } 2001[40]\end{array}$ & $\begin{array}{l}\text { Chinese Taiwanese } \\
\text { patients with VTE }\end{array}$ & no & no & $82(77)$ & 61 & $\begin{array}{l}\text { DVT } \\
\text { or PE }\end{array}$ & yes & $\begin{array}{l}\text { patients without a history of VTE } \\
\text { or CHD }\end{array}$ & $82(77)$ & 61 \\
\hline $\begin{array}{l}\text { Ray } \\
\text { et al. } 2001[41]\end{array}$ & $\begin{array}{l}\text { Canadian women with } \\
\text { VTE and no CVD }\end{array}$ & no & no & $0(0)$ & 46 & $\begin{array}{l}\text { DVT } \\
\text { or PE }\end{array}$ & yes & $\begin{array}{l}\text { healthy women without a history } \\
\text { of VTE or CVD }\end{array}$ & $0(0)$ & 41 \\
\hline
\end{tabular}

Figures in parentheses represent percentages. NA = Not available; DVT = deep vein thrombosis; $\mathrm{PE}=$ pulmonary embolism;

$\mathrm{CHD}=$ coronary heart disease $\mathrm{CVD}=$ cardiovascular disease.

1 Defined as a deficiency of either protein C, protein S or antithrombin or the presence of antiphospholipid antibodies. 


\section{Data Abstraction}

Results were independently abstracted by two authors (W.S.C. and J.G.R.), as listed in tables $1-3$. We collected data on the absolute number of individuals with and without VTE who had the MTHFR C677T wild-type (CC), heterozygous (CT) or homozygous (TT) genotypes (tables 2, 3). Again, authors were contacted by E-mail if the latter figures were incomplete. We also abstracted information about the methods used to recruit the cases and controls, and whether there was exclusion of those with VTE related to surgery, trauma or cancer or a known 'classic' thrombophilia factor (i.e. a deficiency of either protein $\mathrm{C}$, protein $\mathrm{S}$ or antithrombin or the presence of antiphospholipid antibodies). Finally, we assessed whether cases were diagnosed with VTE using objective diagnostic methods, such as compression ultrasonography for deep vein thrombosis of the limb and ventilation-perfusion scanning for pulmonary embolism.

\section{Statistical Analysis}

In the primary analysis, the rate of the MTHFR C677T TT genotype was calculated among cases and controls and compared to the presence of either the CT or CC genotype. An unadjusted OR and its $95 \%$ CI was calculated for each study, and then pooled $\left(\mathrm{OR}_{\mathrm{p}}\right)$ using a random effects model, which considers both between-study and within-study variability [9]. The prevalence rates of the TT genotype among cases and controls were pooled using the same random effects method. A second analysis excluded those studies that did not explicitly describe the use of objective testing methods to diagnose VTE.

A third and fourth analysis comprised only those studies that systematically excluded cases with malignancy or VTE in the presence of surgery or trauma (i.e. situational VTE), as well as a 'classic' thrombophilia factor. Heterogeneity across studies was assessed using the Breslow and Day [10] test, with statistical significance set at $\mathrm{p}<0.10$. The applied statistical software program used was MetaAnalyst ${ }^{0.988}[11]$.

Table 2. Number and rate (in parentheses) of the C677T MTHFR genotypes and the risk of VTE from all 31 studies

\begin{tabular}{|c|c|c|c|c|c|c|c|c|}
\hline \multirow[t]{2}{*}{ Study } & \multicolumn{4}{|c|}{ Cases with VTE } & \multicolumn{4}{|c|}{ Controls without VTE } \\
\hline & $\mathrm{CC}$ & $\mathrm{CT}$ & TT & total & $\mathrm{CC}$ & $\mathrm{CT}$ & TT & total \\
\hline Arruda et al., 1997 [7] & $61(48)$ & $52(41)$ & $14(11)$ & $127(100)$ & $170(58)$ & $114(38)$ & $12(4.0)$ & $296(100)$ \\
\hline Cattaneo et al., 1997 [12] & $25(32)$ & $36(47)$ & $16(21)$ & $77(100)$ & $45(29)$ & $74(48)$ & $35(23)$ & $154(100)$ \\
\hline Ocal et al., 1997 [13] & $139(42)$ & $154(47)$ & $38(11)$ & $331(100)$ & $62(37)$ & $79(47)$ & $26(16)$ & $167(100)$ \\
\hline Salden et al., 1997 [14] & $88(41)$ & $102(47)$ & $26(12)$ & $216(100)$ & $71(43)$ & $75(46)$ & $18(11)$ & $164(100)$ \\
\hline Tosetto et al., 1997 [15] & $21(32)$ & $36(55)$ & $8(12)$ & $65(100)$ & $42(32)$ & $71(55)$ & $17(13)$ & $130(100)$ \\
\hline Brown et al., 1998 [16] & $261(47)$ & $242(43)$ & $55(10)$ & $558(100)$ & $224(45)$ & $209(42)$ & $67(13)$ & $500(100)$ \\
\hline Dilley et al., 1998 [17] & $77(83)$ & $15(16)$ & $1(1)$ & $93(100)$ & $152(82)$ & $29(16)$ & $4(2)$ & $185(100)$ \\
\hline Grandone et al., 1998 [18] & $12(29)$ & $18(43)$ & $12(29)$ & $42(100)$ & $69(32)$ & $110(52)$ & $34(16)$ & $213(100)$ \\
\hline Kluijtmans et al., 1998 [19] & $211(45)$ & $213(45)$ & $47(10)$ & $471(100)$ & $224(47)$ & $203(43)$ & $47(10)$ & $474(100)$ \\
\hline Margaglione et al., 1998 [20] & $67(30)$ & $95(43)$ & $60(27)$ & $222(100)$ & $212(49)$ & $141(33)$ & $78(18)$ & $431(100)$ \\
\hline Rintelen et al., 1999 [21] & $31(38)$ & $40(49)$ & $10(12)$ & $81(100)$ & $35(45)$ & $32(42)$ & $10(13)$ & $77(100)$ \\
\hline Alhenc-Gelas et al., 1999 [22] & $91(44)$ & $88(43)$ & $26(13)$ & $205(100)$ & $156(39)$ & $193(49)$ & $49(12)$ & $398(100)$ \\
\hline Cattaneo et al., 1999 [23] & $33(29)$ & $56(50)$ & $23(21)$ & $112(100)$ & $138(34)$ & $181(45)$ & $84(21)$ & $403(100)$ \\
\hline Ho, $2000[24]$ & $24(49)$ & $17(35)$ & $8(16)$ & $49(100)$ & $583(49)$ & $472(40)$ & $125(11)$ & $1,180(100)$ \\
\hline Franco et al., 1999 [25] & $71(37)$ & $82(43)$ & $37(20)$ & $190(100)$ & $81(43)$ & $78(41)$ & $31(16)$ & $190(100)$ \\
\hline Gemmati et al., 1999 [26] & $51(23)$ & $107(49)$ & $62(28)$ & $220(100)$ & $65(30)$ & $116(53)$ & $39(18)$ & $220(100)$ \\
\hline Gemmati et al., 1999 [27] & $53(29)$ & $86(48)$ & $41(23)$ & $180(100)$ & $68(34)$ & $102(51)$ & $30(15)$ & $200(100)$ \\
\hline Salomon et al., 1999 [28] & $56(34)$ & $69(43)$ & $37(23)$ & $162(100)$ & $136(40)$ & $152(45)$ & $48(14)$ & $336(100)$ \\
\hline Lin 2000 [29] & $53(47)$ & $50(45)$ & $9(8)$ & $112(100)$ & $76(61)$ & $41(33)$ & $8(6)$ & $125(100)$ \\
\hline Akar et al., 2000 [30] & $30(44)$ & $33(48)$ & $5(8)$ & $68(100)$ & $40(61)$ & $19(29)$ & $7(10)$ & $66(100)$ \\
\hline Angchaisuksiri et al., 2000 [31] & $34(85)$ & $6(15)$ & $0(0)$ & $40(100)$ & $365(73)$ & $128(26)$ & $7(1)$ & $500(100)$ \\
\hline Couturaud et al., 2000 [32] & $233(64)$ & $102(28)$ & $31(8)$ & $366(100)$ & $59(56)$ & $36(34)$ & $10(10)$ & $105(100)$ \\
\hline De Franchis et al., 2000 [33] & $88(46)$ & $58(30)$ & $46(24)$ & $192(100)$ & $112(46)$ & $50(24)$ & $42(20)$ & $204(100)$ \\
\hline Fujimura et al., 2000 [34] & $32(44)$ & $30(42)$ & $10(14)$ & $72(100)$ & $36(42)$ & $43(51)$ & $6(7)$ & $85(100)$ \\
\hline Gerhardt et al., 2000 [35] & $50(44)$ & $53(46)$ & $11(10)$ & $114(100)$ & $101(48)$ & $91(43)$ & $20(9)$ & $212(100)$ \\
\hline Toydemir et al., 2000 [36] & $15(50)$ & $12(40)$ & $3(10)$ & $30(100)$ & $39(39)$ & $51(51)$ & $10(10)$ & $100(100)$ \\
\hline Zhang et al., 2000 [37] & $12(23)$ & $31(58)$ & $10(19)$ & $53(100)$ & $62(51)$ & $45(37)$ & $15(12)$ & $122(100)$ \\
\hline Guedon et al., 2001 [38] & $45(41)$ & $52(47)$ & $13(12)$ & $110(100)$ & $32(38)$ & $43(51)$ & $9(11)$ & $84(100)$ \\
\hline Hanson et al., 2001 [30] & $58(42)$ & $63(46)$ & $16(12)$ & $137(100)$ & $130(40)$ & $158(48)$ & $41(12)$ & $329(100)$ \\
\hline Hsu et al., 2001 [40] & $60(57)$ & $40(37)$ & $7(6)$ & $107(100)$ & $55(51)$ & $44(41)$ & $8(8)$ & $107(100)$ \\
\hline Ray et al., 2001 [41] & $49(38)$ & $61(47)$ & $19(15)$ & $129(100)$ & $72(56)$ & $44(34)$ & $13(10)$ & $129(100)$ \\
\hline Total number of individuals studied & \multicolumn{4}{|l|}{4,901} & \multicolumn{4}{|l|}{7,886} \\
\hline Pooled prevalence of TT genotype, $\%$ & \multicolumn{4}{|c|}{$14.3(95 \%$ CI $12.0-16.9)$} & \multicolumn{4}{|c|}{$11.7(95 \%$ CI $10.0-13.5)$} \\
\hline $\begin{array}{l}\mathrm{OR}_{\mathrm{p}} \text { for VTE in the presence of the TT } \\
\text { versus CC or CT genotype }\end{array}$ & \multicolumn{8}{|c|}{$1.2(95 \%$ CI $1.1-1.4)$} \\
\hline \multicolumn{9}{|l|}{$\begin{array}{l}\text { Presence of heterogeneity for the } \\
\qquad \mathrm{OR}_{n}(\mathrm{p}<0.10) \text { ? }\end{array}$} \\
\hline
\end{tabular}




\section{Results}

\section{Study and Participant Characteristics}

A total of 73 citations were retrieved on PubMed Medline and 12 through Embase. Thirty-one original studies from 16 countries met the inclusion criteria (tables 1, 2). The demographic characteristics of the cases and controls are listed in table 1. Most studies had an equal representation of men and women, but 3 studies included only women $[18,35,41]$. The mean age of both cases and controls was 45 years; the majority were aged $40-50$ years.

\section{MTHFR C677T Homozygosity and VTE Risk among All Study Participants}

Only 4 of the 31 studies independently demonstrated a significant association between the MTHFR 677 homozygous state and VTE [7, 18, 20, 26] (fig. 1). The pooled prevalence of the MTHFR 677 TT genotype was higher among the 4,901 cases $(14.3 \%, 95 \%$ CI $12.0-16.9 \%)$ than the 7,886 unaffected controls $(11.7 \%, 95 \%$ CI 10.0 $13.5 \%)\left(\mathrm{OR}_{\mathrm{p}} 1.2,95 \%\right.$ CI 1.1-1.4) (table 2; fig. 1). There was, however, evidence of statistical heterogeneity for this pooled estimate $(\mathrm{p}<0.10)$.
Secondary Analyses of MTHFR C677T Homozygosity and VTE Risk

After excluding 3 studies that did not report on the use of objective VTE testing [13, 38, 39], the associated risk for VTE in the presence of MTHFR 677 TT was not altered substantially from that observed in the main analysis $\left(\mathrm{OR}_{\mathrm{p}} 1.3,95 \% \mathrm{CI} 1.1-1.6\right)$. An analysis of the 14 studies that excluded individuals with VTE in the presence of malignancy or situational risk factors (table 1) also showed no difference in the risk for VTE $\left(\mathrm{OR}_{\mathrm{p}} 1.4,95 \%\right.$ CI 1.2-1.6). Finally, 11 studies formally excluded cases identified as having a 'classic' thrombophilia factor (i.e. protein $\mathrm{C}$, protein $\mathrm{S}$ or antithrombin deficiency or the presence of antiphospholipid antibodies) (tables 1, 3). For these studies, the pooled prevalence of the MTHFR 677 TT genotype was 17.7 (95\% CI 13.7-22.5\%) among 1,171 cases and $12.3 \%(95 \% \mathrm{CI} 9.5-15.7 \%)$ among 2,231 controls $\left(\mathrm{OR}_{\mathrm{p}} 1.5,95 \% \mathrm{CI} 1.2-1.9\right)$. There was no evidence of statistical heterogeneity for any of these secondary analy$\operatorname{ses}(p>0.10)$.

Table 3. Number and rate (in parentheses) of the C677T MTHFR genotypes and the risk of VTE after the exclusion of cases with 'classic' thrombophilia factors

\begin{tabular}{|c|c|c|c|c|c|c|c|c|}
\hline \multirow[t]{2}{*}{ Study } & \multicolumn{4}{|c|}{ Cases with VTE } & \multicolumn{4}{|c|}{ Controls without VTE } \\
\hline & $\mathrm{CC}$ & $\mathrm{CT}$ & TT & total & $\mathrm{CC}$ & $\mathrm{CT}$ & TT & total \\
\hline Arruda et al., 1997 [7] & $49(49)$ & $41(41)$ & $10(10)$ & $100(100)$ & $170(58)$ & $114(38)$ & $12(4)$ & $296(100)$ \\
\hline Salden et al., 1997 [14] & $63(41)$ & $72(47)$ & $17(12)$ & $216(100)$ & $71(43)$ & $75(46)$ & $18(11)$ & $164(100)$ \\
\hline Margaglione et al., 1998 [20] & NA & NA & $28(24)$ & $119(100)$ & NA & NA & $78(18)$ & $431(100)$ \\
\hline Rintelen et al., 1999 [21] & $31(38)$ & $40(49)$ & $10(12)$ & $81(100)$ & $35(45)$ & $32(42)$ & $10(13)$ & $77(100)$ \\
\hline Gemmati et al., 1999 [26] & $51(23)$ & $107(49)$ & $62(28)$ & $220(100)$ & $65(30)$ & $116(53)$ & $39(18)$ & $220(100)$ \\
\hline Gemmati et al., 1999 [27] & $11(22)$ & $24(48)$ & $15(30)$ & $50(100)$ & $62(33)$ & $100(53)$ & $27(14)$ & $189(100)$ \\
\hline Salomon et al. 1999 [28] & NA & NA & $10(12)$ & $80(100)$ & NA & NA & $46(14)$ & $336(100)$ \\
\hline Lin et al., 2000 [29] & $19(36)$ & $26(50)$ & $7(14)$ & $52(100)$ & $64(60)$ & $37(35)$ & $6(6)$ & $107(100)$ \\
\hline De Franchis et al., 2000 [33] & $88(46)$ & $58(30)$ & $46(24)$ & $192(100)$ & $112(46)$ & $50(24)$ & $42(20)$ & $204(100)$ \\
\hline Fujimara et al., $2000[34]$ & $19(40)$ & $25(52)$ & $4(8)$ & $48(100)$ & $36(42)$ & $43(51)$ & $6(7)$ & $85(100)$ \\
\hline Zheng et al., 2000 [37] & $12(23)$ & $31(58)$ & $10(19)$ & $53(100)$ & $62(51)$ & $45(37)$ & $15(12)$ & $122(100)$ \\
\hline Total number of individuals studied & \multicolumn{4}{|l|}{1,171} & \multicolumn{4}{|l|}{2,231} \\
\hline Pooled prevalence of TT genotype, $\%$ & \multicolumn{4}{|c|}{17.7 (95\% CI 13.7-22.5) } & \multicolumn{4}{|c|}{$12.3(95 \%$ CI $9.5-15.7)$} \\
\hline \multicolumn{9}{|l|}{$\begin{array}{l}\mathrm{OR}_{\mathrm{p}} \text { for } \mathrm{VTE} \text { in the presence of the } \mathrm{TT} \\
\text { versus CC or CT genotype }\end{array}$} \\
\hline \multicolumn{9}{|l|}{ Presence of heterogeneity for the } \\
\hline
\end{tabular}




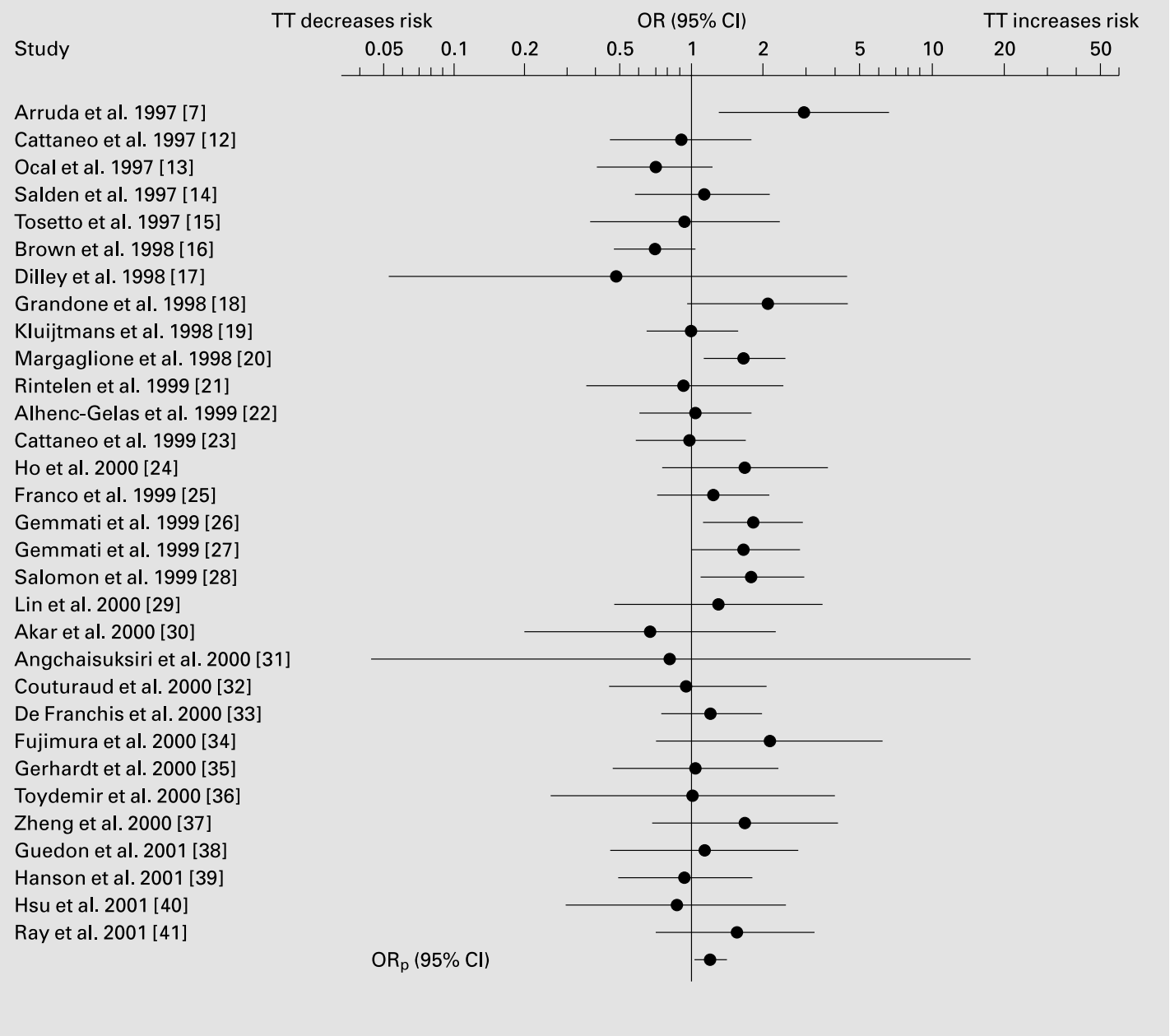

Fig. 1. MTHFR 677 TT versus CC or CT genotypes and the risk of VTE.

\section{Discussion}

\section{Principal Findings}

We examined 31 published studies, comprising nearly 13,000 individuals, and demonstrated a weak association between the MTHFR 677 TT genotype and VTE. This relationship was only slightly more impressive after excluding individuals with malignancy or situational VTE or a classic thrombophilia factor.

\section{Strengths and Weaknesses}

A major strength of this review was the large number of individuals and participating countries included herein. Most studies contained at least 50 cases with objectively confirmed deep vein thrombosis or pulmonary embolism, the majority being middle aged and without malignancy or situational risk factors for VTE.

Such features make these data applicable to many patients with idiopathic VTE in whom a thrombophilia workup might be considered. Had publication bias been a major factor herein, then it is likely that the observed effect size would have been deflated further had unpublished studies been included as well [42].

\section{Sources of Heterogeneity}

The presence of statistical heterogeneity detected in the primary analysis of all 31 studies might be explained, in part, by the different methods used to recruit cases and controls. For example, some cases were enlisted at the time of their initial hospitalization for VTE, while others 
were referred to a specialized clinic at a later date. Similarly, study controls were a heterogeneous group of individuals, ranging from newborn infants [7] to hospitalized adults [28]. The variety of countries included herein is another likely source of heterogeneity, since ethnogeographic differences in the prevalence of the MTHFR C677T polymorphism have been demonstrated $[43,44]$.

\section{Clinical Considerations}

Given the weak direct relationship between the MTHFR C677T polymorphism and VTE, we do not believe that this test should be routinely included in any clinical thrombophilia workup until more compelling data are made available. Our data parallel those of Brattstrom et al. [6], whose meta-analysis failed to demonstrate an association between MTHFR C677T homozygosity and arterial disease $\left(\mathrm{OR}_{\mathrm{p}} 1.1,95 \% \mathrm{CI} 0.9-1.4\right)$. Since we did not assess the influence of the MTHFR C677T polymorphism in conjunction with either folate or cobalamin deficiency or in the presence of other genetic thrombophilia factors [45], we cannot exclude a possible role therein [44]. However, we recently observed that none of three MTHFR polymorphisms (C677T, A1298C and $\mathrm{T} 1317 \mathrm{C}$ ), nor the A66G polymorphism in methionine synthase reductase, were demonstrable risk factors for VTE, either alone or in conjunction with fasting plasma hyperhomocysteinemia [41].

\section{Future Research}

The purported positive relationship between elevated tHcy and VTE [1] requires further explication beyond the MTHFR C677T polymorphism. The current challenge is to understand which other gene(s) play a role not only in the elevation of tHcy, but also in the development of idiopathic VTE. A large prospective population-based cohort study [46] is one appropriate way to evaluate both the environmental and multigenic factors that predispose to VTE [47].

\section{References}

1 Ray JG: Meta-analysis of hyperhomocysteinemia as a risk factor for venous thromboembolic disease. Arch Intern Med 1998;158: 2101-2106.

2 Kang SS, Wong PW, Zhou JM, Sora J, Lessick M, Ruggie N, Grcevich G: Thermolabile methylenetetrahydrofolate reductase in patients with coronary artery disease. Metabolism 1988;37:611-613.

3 Rozen R: Genetic modulation of homocysteinemia. Semin Thromb Hemost 2000;26:255261.

4 Boushey CJ, Beresford SA, Omenn GS, Motulsky AG: A quantitative assessment of plasma homocysteine as a risk factor for vascular disease. Probable benefits of increasing folic acid intakes. JAMA 1995;274:1049-1057.

5 Graham IM, Daly LE, Refsum HM, Robinson K, Brattstrom LE, Ueland PM, Palma-Reis RJ, Boers GH, Sheahan RG, Israelsson B, Uiterwaal CS, Meleady R, McMaster D, Verhoef P, Witteman J, Rubba P, Bellet H, Wautrecht JC, de Valk HW, Sales Luis AC, Parrot-Rouland FM, Tan KS, Higgins I, Garcon D, Andria G Plasma homocysteine as a risk factor for vascular disease. The European Concerted Action Project. JAMA 1997;277:1775-1781.

6 Brattstrom L, Wilcken DE, Ohrvik J, Brudin L: Common methylenetetrahydrofolate reductase gene mutation leads to hyperhomocysteinemia but not to vascular disease: The result of a meta-analysis. Circulation 1998;98:2520 2526

MTHFR Gene Polymorphism and Venous

Thromboembolism
7 Arruda VR, von Zuben PM, Chiaparini LC, Annichino-Bizzacchi JM, Costa FF: The mutation Ala677 $\rightarrow$ Val in the methylene tetrahydrofolate reductase gene: A risk factor for arterial disease and venous thrombosis. Thromb Haemost 1997;77:818-821.

8 De Stefano V, Casorelli I, Rossi E, Zappacosta B, Leone G: Interaction between hyperhomocysteinemia and inherited thrombophilic factors in venous thromboembolism. Semin Thromb Hemost 2000;26:305-311.

9 DerSimonian R, Laird N: Meta-analysis in clinical trials. Control Clin Trials 1986;7:177188.

10 Breslow NE, Day NE: Statistical methods in cancer research. Volume I - The analysis of case-control studies. IARC Sci Publ 1980;32: 5-338.

11 Lau J: Meta-Analyst ${ }^{0.998}$ Statistical Software. Boston, 1995.

12 Cattaneo M, Tsai MY, Bucciarelli P, Taioli E, Zighetti ML, Bignell M, Mannucci PM: A common mutation in the methylenetetrahydrofolate reductase gene (C677T) increases the risk for deep-vein thrombosis in patients with mutant factor V (factor V:Q506). Arterioscler Thromb Vasc Biol 1997; 17:1662-1666.

13 Ocal IT, Sadeghi A, Press RD: Risk of venous thrombosis in carriers of a common mutation in the homocysteine regulatory enzyme methylenetetrahydrofolate reductase. Mol Diagn 1997;2:61-68

14 Salden A, Keeney S, Hay CRM, Cumming AM: The C677T MTHFR variant and the risk of venous thrombosis. Br J Haematol 1997;99: 472 .
15 Tosetto A, Missiaglia E, Frezzato M, Rodeghiero F: The VITA project: C677T mutation in the methylene-tetrahydrofolate reductase gene and risk of venous thromboembolism. Br J Haematol 1997;97:804-806.

16 Brown K, Luddington R, Baglin T: Effect of the MTHFRC677T variant on risk of venous thromboembolism: Interaction with factor $\mathrm{V}$ Leiden and prothrombin (F2G20210A) mutations. Br J Haematol 1998;103:42-44.

17 Dilley A, Austin H, Hooper WC, Lally C, Ribeiro MJ, Wenger NK, Silva V, Rawlins P, Evatt B: Relation of three genetic traits to venous thrombosis in an African-American population. Am J Epidemiol 1998;147:30-35.

18 Grandone E, Margaglione M, Colaizzo D, D'Andrea G, Cappucci G, Brancaccio V, Di Minno G: Genetic susceptibility to pregnancyrelated venous thromboembolism: Roles of factor V Leiden, prothrombin G20210A, and methylenetetrahydrofolate reductase C677T mutations. AM J Obstet Gynecol 1998;179: 1324-1328.

19 Kluijtmans LA, den Heijer M, Reitsma PH Heil SG, Blom HJ, Rosendaal FR: Thermolabile methylenetetrahydrofolate reductase and factor V Leiden in the risk of deep-vein thrombosis. Thromb Haemost 1998;79:254-258.

20 Margaglione M, D'Andrea G, d'Addedda M, Giuliani N, Cappucci G, Iannaccone L, Vecchione G, Grandone E, Brancaccio V, Di Minno G: The methylenetetrahydrofolate reductase TT677 genotype is associated with venous thrombosis independently of the coexistence of the FV Leiden and the prothrombin A20210 mutation. Thromb Haemost 1998;79:907911. 
21 Rintelen C, Mannhalter C, Lechner K, Eichinger S, Kyrle PA, Papagiannopoulos M Schneider B, Pabinger I: No evidence for an increased risk of venous thrombosis in patients with factor V Leiden by the homozygous $677 \mathrm{C}$ to $\mathrm{T}$ mutation in the methylenetetrahydrofolate-reductase gene. Blood Coagul Fibrinolysis 1999;10:101-105.

22 Alhenc-Gelas M, Arnaud E, Nicaud V, Aubry ML, Fiessinger JN, Aiach M, Emmerich J: Venous thromboembolic disease and the prothrombin, methylene tetrahydrofolate reductase and factor $\mathrm{V}$ genes. Thromb Haemost 1999;81:506-511.

23 Cattaneo M, Chantarangkul V, Taioli E, Santos JH, Tagliabue L: The G20210A mutation of the prothrombin gene in patients with previous first episodes of deep-vein thrombosis: Prevalence and association with factor V G1691A, methylenetetrahydrofolate reductase C677T and plasma prothrombin levels. Thromb Res 1999;93:1-8.

24 Ho CH: Prevalence of prothrombin 20210A allele and methylenetetrahydrofolate reductase C677T genetic mutations in the Chinese population. Ann Hematol 2000;79:239-242.

25 Franco RF, Morelli V, Lourenco D, Maffei FH, Tavella MH, Piccinato CE, Thomazini IA, Zago MA: A second mutation in the methylenetetrahydrofolate reductase gene and the risk of venous thrombotic disease. Br J Haematol 1999; 105:556-559.

26 Gemmati D, Previati M, Serino ML, Moratelli S, Guerra S, Capitani S, Forini E, Ballerini G, Scapoli GL: Low folate levels and thermolabile methylenetetrahydrofolate reductase as primary determinant of mild hyperhomocysteinemia in normal and thromboembolic subjects. Arterioscler Thromb Vasc Biol 1999;19:17611767.

27 Gemmati D, Serino ML, Trivellato C, Fiorini $\mathrm{S}$, Scapoli GL: C677T substitution in the methylenetetrahydrofolate reductase gene as a risk factor for venous thrombosis and arterial disease in selected patients. Haematologica 1999 84:824-828.

28 Salomon O, Steinberg DM, Zivelin A, Gitel S, Dardik R, Rosenberg N, Berliner S, Inbal A Many A, Lubetsky A, Varon D, Martinowitz U, Seligsohn U: Single and combined prothrombotic factors in patients with idiopathic venous thromboembolism: Prevalence and risk assessment. Arterioscler Thromb Vasc Biol 1999;19. 511-518.
29 Lin JS, Shen MC, Tsai W: The prevalence of C677T mutation in the methylenetetrahydrofolate reductase gene and its association with venous thrombophilia in Taiwanese Chinese. Thromb Res 2000;97:89-94.

30 Akar N, Akar E, Akcay R, Avcu F, Yalcin A, Cin S: Effect of methylenetetrahydrofolate reductase 677 C-T, 1298 A-C, and 1317 T-C on factor V 1691 mutation in Turkish deep vein thrombosis patients. Thromb Res 2000;97: 163-167.

31 Angchaisuksiri P, Pingsuthiwong S, Sura T, Aryuchai K, Busabaratana M, Atichartakarn V: Prevalence of the C677T methylenetetrahydrofolate reductase mutation in Thai patients with deep vein thrombosis. Acta Haematol 2000;103:191-196.

32 Couturaud F, Oger E, Abalain JH, Chenu E, Guias B, Floch HH, Mercier B, Mottier D, Leroyer C: Methylenetetrahydrofolate reductase C677T genotype and venous thromboembolic disease. Respiration 2000;67:657-661.

33 de Franchis R, Fermo I, Mazzola G, Sebastio G, Di Minno G, Coppola A, Andria G, D’Angelo A: Contribution of the cystathionine betasynthase gene (844ins68) polymorphism to the risk of early-onset venous and arterial occlusive disease and of fasting hyperhomocysteinemia. Thromb Haemost 2000;84:576-582.

34 Fujimura H, Kawasaki T, Sakata T, Ariyoshi $\mathrm{H}$, Kato H, Monden M, Miyata T: Common C677T polymorphism in the methylenetetrahydrofolate reductase gene increases the risk for deep vein thrombosis in patients with predisposition of thrombophilia. Thromb Res 2000;98:1-8.

35 Gerhardt A, Scharf RE, Beckmann MW, Struve S, Bender HG, Pillny M, Sandmann W, Zotz RB: Prothrombin and factor V mutations in women with a history of thrombosis during pregnancy and the puerperium. N Engl J Med 2000;342:374-380

36 Toydemir PB, Elhan AH, Tukun A, Toydemir R, Gurler A, Tuzuner A, Bokesoy I: Effects of factor V gene G1691A, methylenetetrahydrofolate reductase gene $\mathrm{C} 677 \mathrm{~T}$, and prothrombin gene G20210A mutations on deep venous thrombogenesis in Behcet's disease. J Rheumatol 2000;27:2849-2854.

37 Zheng YZ, Tong J, Do XP, Pu XQ, Zhou BT: Prevalence of methylenetetrahydrofolate reductase C677T and its association with arterial and venous thrombosis in the Chinese population. Br J Haematol 2000;109:870-874.
38 Guedon C, Le Cam-Duchez V, Lalaude O, Menard JF, Lerebours E, Borg JY: Prothrombotic inherited abnormalities other than factor $\mathrm{V}$ Leiden mutation do not play a role in venous thrombosis in inflammatory bowel disease. Am J Gastroenterol 2001;96:1448-1454.

39 Hanson NQ, Aras O, Yang F, Tsai MY: C677T and A1298C polymorphisms of the methylenetetrahydrofolate reductase gene: Incidence and effect of combined genotypes on plasma fasting and post-methionine load homocysteine in vascular disease. Clin Chem 2001;47:661-666.

40 Hsu LA, Ko YL, Wang SM, Chang CJ, Hsu TS Chiang CW, Lee YS: The C677T mutation of the methylenetetrahydrofolate reductase gene is not associated with the risk of coronary artery disease or venous thrombosis among Chinese in Taiwan. Hum Hered 2001;51:4145.

41 Ray JG, Langman LJ, Vermeulen MJ, Evrovski J, Yeo EL, Cole DE: Genetics University of Toronto Thrombophilia Study in Women (GUTTSI): Genetic and other risk factors for venous thromboembolism in women. Curr Control Trials Cardiovasc Med 2001;2:141149 .

42 Sutton AJ, Song F, Gilbody SM, Abrams KR: Modelling publication bias in meta-analysis: A review. Stat Methods Med Res 2000;9:421445.

43 Hessner MJ, Luhm RA, Pearson SL, Endean DJ, Friedman KD, Montgomery RR: Prevalence of prothrombin G20210A, factor V G1691A (Leiden), and methylenetetrahydrofolate reductase (MTHFR) C677T in seven different populations determined by multiplex allele-specific PCR. Thromb Haemost 1999;81: 733-738.

44 Mager A: Methylenetetrahydrofolate reductase gene and coronary artery disease. Circulation 2000;101:E172-E173.

45 Zoller B, Garcia de Frutos P, Hillarp A, Dahlback B: Thrombophilia as a multigenic disease. Haematologica 1999;84:59-70.

46 Nygard O, Vollset SE, Refsum H, Stensvold I, Tverdal A, Nordrehaug JE, Ueland M, Kvale G: Total plasma homocysteine and cardiovascular risk profile. The Hordaland Homocysteine Study. JAMA 1995;274:1526-1533.

47 Ehrenforth S, Junker R, Koch HG, Kreuz W, Munchow N, Scharrer I, Nowak-Gottl U: Multicentre evaluation of combined prothrombotic defects associated with thrombophilia in childhood. Childhood Thrombophilia Study Group Eur J Pediatr 1999;158(suppl 3):S97-S104. 